\title{
Utility of transthoracic echocardiography (TTE) in assessing fluid responsiveness in critically ill patients - a challenge for the bedside sonographer
}

\author{
Wojciech Mielnicki, Agnieszka Dyla, Tomasz Zawada
}

$4^{\text {th }}$ Military Clinical Hospital, Wroclaw, Poland

\begin{abstract}
Transthoracic echocardiography (TTE) has become one of the most important diagnostic tools in the treatment of critically ill patients. It allows clinicians to recognise potentially reversible life-threatening situations and is also very effective in the monitoring of the fluid status of patients, slowly substituting invasive methods in the intensive care unit. Hemodynamic assessment is based on a few static and dynamic parameters. Dynamic parameters change during the respiratory cycle in mechanical ventilation and the level of this change directly corresponds to fluid responsiveness. Most of the parameters cannot be used in spontaneously breathing patients. For these patients the most important test is passive leg raising, which is a good substitute for fluid bolus. Although TTE is very useful in the critical care setting, we should not forget the important limitations, not only technical ones but also caused by the critical illness itself. Unfortunately, this method does not allow continuous monitoring and every change in the patient's condition requires repeated examination.
\end{abstract}

Keywords: hypovolaemia, non-invasive monitoring, intensive care unit, transthoracic echocardiography

\section{Introduction}

In certain clinical situations, such as shock, hypotension or renal failure, it is necessary to determine the fluid status of the patient to guide fluid therapy. Both hypovolemia, which might be suspected in shock, and hypervolemia resulting from fluid overload, can cause the patient's deterioration and eventually lead to death $[1,2]$. Giving the right amount of fluid to the right patient would be the best solution, but how to choose such a patient?

There are two approaches that might be adapted by the intensivist, as far as fluids are concerned. One can perform a fluid challenge [3] or one can predict fluid responsiveness [4] [5]. When performing a fluid challenge, the attending intensivist infuses fluids and analyses the clinical effect in terms of increase in the blood pressure, cardiac output, central venous pressure (CVP),

Received 17.08.2016 Accepted 18.09.2016

Med Ultrason

2016, Vol. 18, No 4, 508-514

Corresponding author: Agnieszka Dyla,

4th Military Clinical Hospital

Weigla 5, 50-981 Wrocław, Poland

Phone: +48667972982

E-mail: dylusia@wp.pl pulmonary artery occlusion pressure (PAOP), and signs of shock and tissue perfusion improvement. Thus the response is given after the treatment. In contrast, with the fluid responsiveness approach, the intensivist analyses and records some indicators before fluid infusion to predict the effect in terms of increasing the cardiac output [6]. Fluid responsiveness assessment can help to select patients who might benefit from fluid therapy. Selecting the so-called "fluid responder" does not mean obligatory fluid therapy. The decision whether to give fluids or not, should be based on a holistic approach.

Fluid responsiveness can be assessed noninvasively by transthoracic echocardiography (TTE) $[7,8]$. TTE, thanks to its non-invasiveness, bears no negative consequences for the patient. The downside is its lack of continuity and every change in patient's condition has to be verified in repeated examination. Also it requires a lot of experience for proper assessment, because the result of the examination can play a major role in the patient's management.

The subject of this educational paper is an overview of the most important parameters measured by transthoracic echocardiography, used to assess fluid responsiveness in the critical care patient namely the spontaneously 
breathing patient and the patient on mechanical ventilation.

\section{Heart-lung interactions during mechanical ventilation.}

During spontaneous breathing (ie, negative intrathoracic pressure), right atrial pressure is low, impedance to the blood flow to the right ventricle is also low, and systemic venous return is normal. Initiation of positive pressure ventilation (invasive or noninvasive) increases intrathoracic pressure, which is transmitted to the right heart (ie, increased right atrial pressure) and can result in a decrease in systemic venous blood return (ie, decreased right heart preload). This effect is most pronounced in situations of significant increases in mean intrathoracic pressure and/or decreases in intravascular volume (ie, septic, hemorrhagic, or hypovolemic shock). In summary, positive pressure ventilation (PPV) increases mean intrathoracic pressure and reduces right ventricular performance by decreasing right ventricular preload [9].

In contrast to the right ventricle, the left ventricle receives blood from within the thorax (ie, right ventricle via the pulmonary circulation) and pumps it outside the thorax $[9,10]$. Thus, the physiological effects of mechanical ventilation on the left ventricle are quite different from those on the right ventricle as described previously [9]. Applying a positive pressure ventilation will compress pulmonary capillaries and will increase right ventricle afterload. But it will also have consequences downstream by pushing blood from the capillaries to the left ventricle, improving left ventricle filling (LV) and left ventricle stoke volume (LVSV) [6]. Decrease in the LV afterload is also thought to explain the improvement in LV ejection during positive pressure ventilation $[6,11,12]$. It could be related to an increase in the intrathoracic pressure $[6,13]$.

\section{Static parameters to assess fluid responsiveness}

A static parameter is a parameter measured under single ventricular loading conditions.

\section{Inferior vena cava (IVC) diameter}

Inferior vena cava (IVC) diameter is visualised in the subcostal view. The measurement is taken 2 centimetres from the right atrium at end expiration. If the patient is mechanically ventilated there is no correlation between the IVC diameter and fluid responsiveness, as demonstrated by Feissel et al. in septic shock patients [14]. However, small IVC $<10 \mathrm{~mm}$ can predict positive response to fluids. In contrast, IVC with a large diameter $(>20 \mathrm{~mm})$ often excluded any response to fluids $[6,14]$.

\section{Evaluation of ventricles}

Analysis of ventricle size and function is one of the staples of echocardiographic examination. "Eyeballing", which is a visual assessment of the heart, is useful in the quick assessment of myocardial function. Of course "eyeballing" is not a perfect tool and can lead to discrepancies between different examiners [15], but in quick decision making situations it plays the most important role.

Assessing LV size and its behaviour during the cardiac cycle is very helpful in determining fluid status in mechanically ventilated patients. Critically ill patients with suspicion of hypovolemia have small and hyperdynamic ventricles in echocardiographic examination [16,17]. It does not mean that every patient with this kind of left cavity will be fluid responsive, but the chances become bigger the smaller the ventricle is. Fluid responsiveness is very probable when the so-called "kissing ventricles", phenomenon caused by end-systolic effacement (fig 1) is seen. Views dedicated for the assessment of "kissing ventricles" are the parasternal long axis view (PLAX) and parasternal short axis view (SAX). The diagnostic tool that is very helpful for the visualisation of "kissing ventricles" is motion mode (M-mode).

Suggestions:

a. Small, hyperdynamic LV might correlate with fluid responsiveness in mechanically ventilated patients.

b. "Kissing ventricles" diagnosed by echocardiography in patients in shock might require fluid bolus as the first line treatment.

\section{Systolic anterior motion (SAM) of the anterior leaflet of the mitral valve}

SAM is a very interesting finding while performing echocardiography. SAM is seen in many clinical situations with impeded flow through the left ventricle outflow tract (LVOT) [18]. In the case of fluid depletion, especially when the left ventricle is small and hyperdynamic, there can be functional LVOT obstruction caused by the movement of anterior mitral valve leaflet towards the septum in systole (fig 2). It is not easy to find while

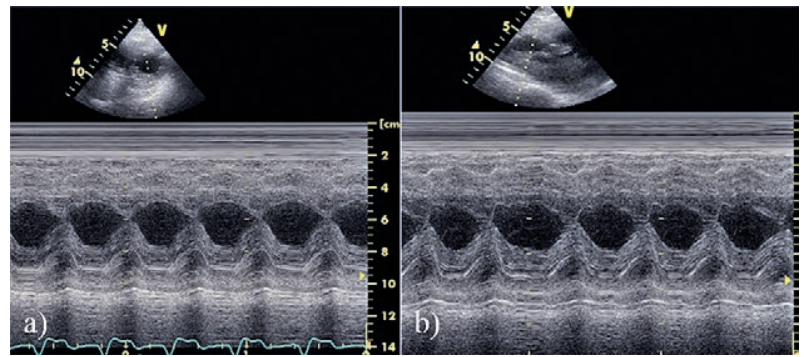

Fig 1. "Eyeballing" of the left ventricle in M-mode. The pictures show „kissing” papillary muscles in a short and long axis view. 
510 Wojciech Mielnicki et al Utility of transthoracic echocardiography (TTE) in assessing fluid responsiveness in critically ill patients

performing quick "eyeballing”. SAM can be visualised in PLAX and five chamber view.

Suggestions:

a. SAM can suggest fluid responsiveness, especially when it is diagnosed as a new finding in a previously healthy patient. Diagnosing requires an experienced echocardiographer.

In conclusion, static parameters are not very useful in predicting fluid responsiveness in spontaneously or mechanically ventilated patients, except in patients with a very low preload.

\section{Dynamic parameters to assess fluid responsiveness}

A dynamic parameter is a parameter that changes during the breathing cycle or fluid infusion, as in the passive leg raising test (PLR).

\section{IVC respiratory variations}

A) Spontaneously breathing patient

During spontaneous breathing IVC collapses during inspiration due to negative intrathoracic pressure. During expiration, when intrathoracic pressure increases

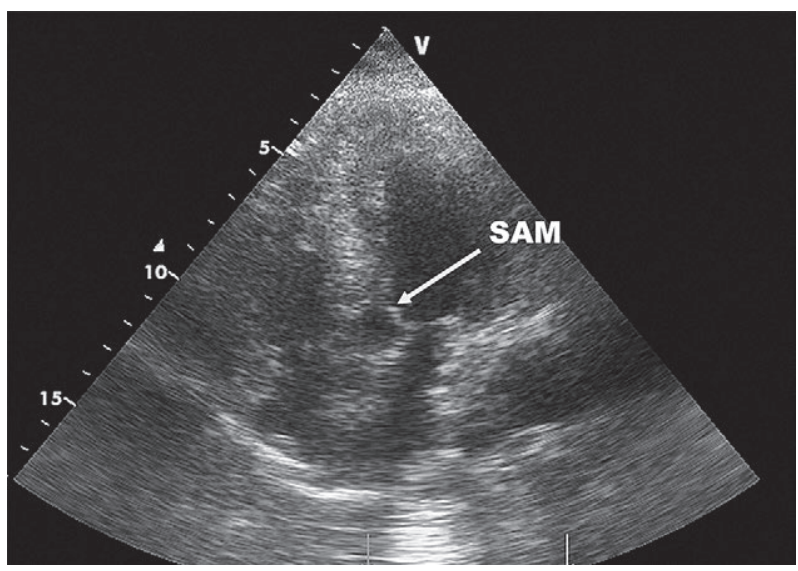

Fig 2. Systolic anterior motion of the mitral valve towards the interventricular septum in the five chamber view.

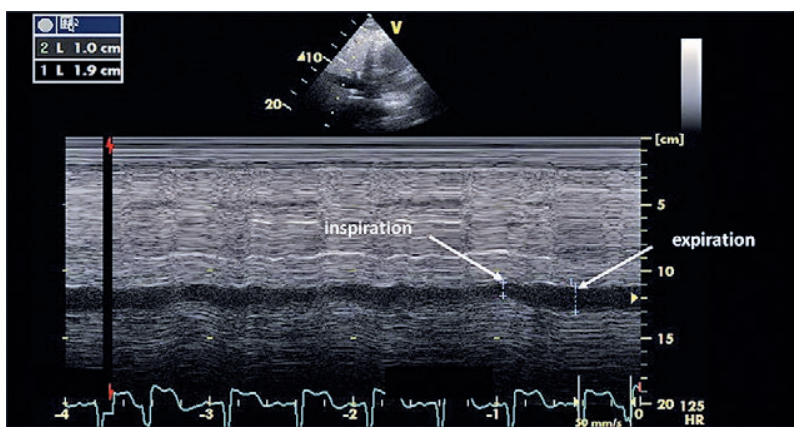

Fig 3. Inferior vena cava collapse during inspiration in a spontaneously breathing patient. to atmospheric pressure IVC diameter also increases. Collapsibility of IVC during spontaneous breathing can be assessed by the so-called "collapsibility index". The "collapsibility index", presented as a percentage, is calculated using the following formula:

$$
\text { Collapsibility index }=\frac{\text { eIVC-iIVC }}{\text { eIVC }} 100 \% \text {, }
$$

where eIVC is the maximal IVC diameter during expiration and iIVC is the minimal IVC diameter during inspiration. It is very difficult to indicate what the exact cut-off value is indicating fluid responsiveness. In recent published papers the percentage was established to be as high as $40-42 \%[19,20]$, but values $<40 \%$ do not rule out positive response to fluid therapy [20] (fig 3). Of course the breathing pattern can affect changes in the IVC diameter especially when the patient presents with dyspnea. In such a patient "collapsibility index" is not reliable at all and IVC changes might be caused by diaphragm compression [21].

Suggestions:

a. There is not enough data supporting using the "collapsibility index" in spontaneously breathing patients as a predictor of fluid responsiveness

b. Latest studies showing good correlation between "collapsibility index" $>40-40 \%$ and positive response to fluids are too small and more data is needed.

\section{B) Mechanically ventilated patient}

In mechanically ventilated patients, during inspiration the pressure in thorax increases due to positive pressure applied by the ventilator. Positive pressure impedes venous return and can cause IVC distension [14]. This phenomenon is the reverse situation to spontaneously breathing patients. IVC distension during mechanical ventilation can be assessed by the so-called "distensibility index". "Distensibility index" can be calculated using one of the two following formulas:

$$
\text { Distensibility index }=\frac{\text { iIVC-eIVC }}{\mathrm{mIVC}} 100 \%
$$

where iIVC is the maximal IVC diameter during inspiration, eIVC is the minimal diameter during inspiration and mIVC is the mean of iIVC and eIVC. For this formula the cut-off value that correlates with fluid responsiveness is $>12 \%$ [14]. The other formula is as follows:

$$
\text { Distensibility index }=\frac{\text { iIVC-eIVC }}{\text { eIVC }} 100 \%
$$

where iIVC is the maximal IVC diameter during inspiration, eIVC is the minimal diameter during expiration. For this formula the cut-off value that correlates with fluid responsiveness is $>18 \%$ [22] (fig 4).

There are some pitfalls with IVC measurement. In addition for the need to obtain a good-quality image, translational artifacts may be a problem. As the respira- 
tory cycles, it displaces liver and adjacent IVC. In such cases, there is no actual change in IVC size, although this is how it appears [6]. Moreover, IVC measurement is very difficult in patients after abdominal surgeries, where achieving a good view is often impossible.

Suggestions:

a. In mechanically ventilated patients IVC "distensibility index" $>12-18 \%$ correlates well with fluid responsiveness.

\section{Velocity-time integral (VTI) variation in left ventricle outflow tract (LVOT) in mechanically ventilated patients}

Left ventricular outflow tract (LVOT) analysis plays a very important role in assessing fluid responsiveness. Flow through the LVOT is the final result of interactions between systemic and pulmonary circulations and pressure in thorax. All medical interventions should be verified by measurements done in LVOT. When a patient is mechanically ventilated, the positive pressure applied causes changes in the tidal volume. Tidal volume and subsequent changes in intrathoracic pressures can influence the volume of blood being pumped through LVOT. The view dedicated for LVOT assessment is a five chamber view. First of all, pulsed wave Doppler (PW) analysis is performed in LVOT (fig 5). It is important to place sampling volume as parallel as possible (within 15 degrees) to the outflow tract to avoid the underestimation of flow. Secondly, stroke volume (SV) or simply velocity-time integral (VTI) is measured. SV measurement requires measurement of LVOT diameter done in PLAX. For hemodynamic purposes VTI measurement is a good option, because any differences in LVOT diameter measurements can lead to big changes in SV.

Respiratory variations of VTI during mechanical ventilation correlate well with fluid responsiveness [23]. Because the respiratory cycle usually lasts a few seconds it is necessary to record all VTIs during the whole cycle. When differences between VTIs reach $\geq 20 \%$, it means the patient might benefit from fluid infusion [17]. Another way to analyse differences in VTI is the measurement of maximal flow velocities in LVOT. Differences in maximal velocities $\geq 12 \%$ in septic shock patients correspond to positive response to fluids [24] (fig 6).

Unfortunately there are many limitations in VTI measurements[25-28]:

- The probe should be in the optimal position and incident angle parallel, near parallel to the LVOT.

- The patient has to be mechanically ventilated in a controlled mode with a tidal volume of at least $8 \mathrm{ml} / \mathrm{kg}$

- The patient cannot breathe spontaneously.

- The patient should be in sinus rhythm.

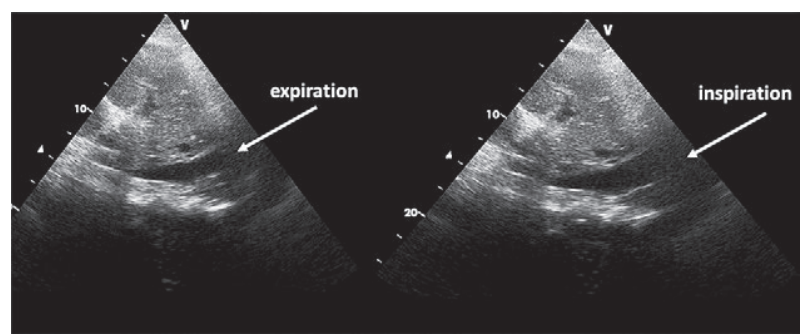

Fig 4. Inferior vena cava distends during inspiration in mechanically ventilated patients. Positive pressure ventilation causes an increase in the inferior vena cava diameter.

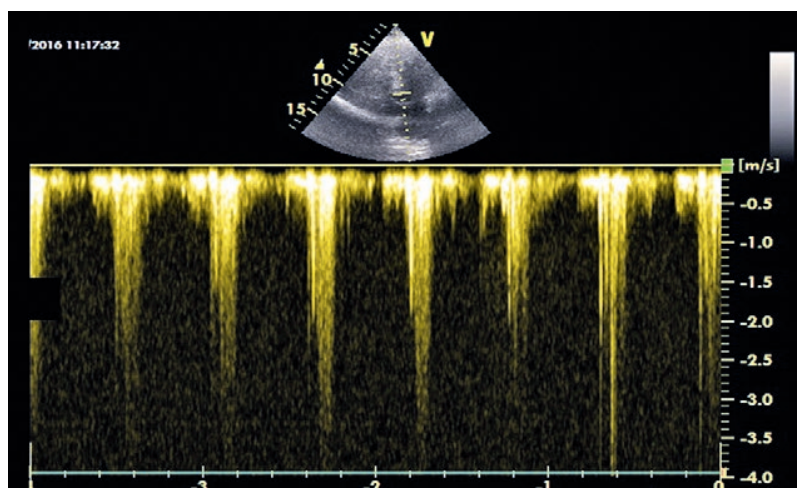

Fig 5. Continuous wave Doppler through the Left ventricular outflow tract shows high velocities ( almost $4 \mathrm{~m} / \mathrm{s}$ ).

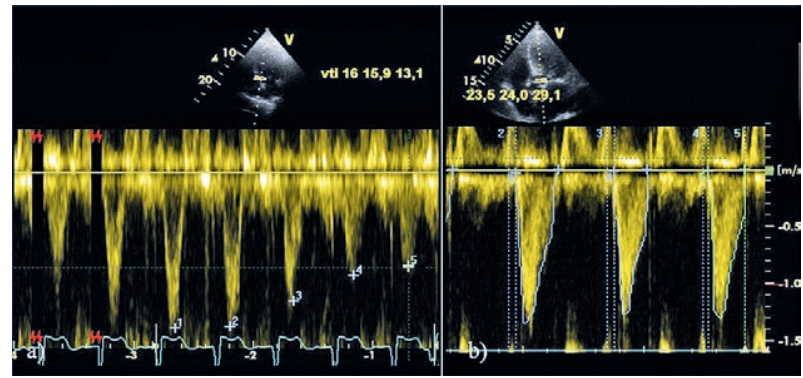

Fig 6. Differences in velocities and velocity-time integrals in mechanically ventilated patients. On the left: difference between the highest maximal velocity of VTI measured in the left ventricle outflow tract (1) and the lowest maximal velocity of VTI in the left ventricle outflow tract (5) exceeds $20 \%$; VTI pattern resembles sinusoid. On the right: difference between the measured minimal VTI (1) and maximal VTI exceeds $12 \%$. These two situations might suggest fluid responsiveness in mechanically ventilated patients.

- Abdominal hypertension should be excluded.

- These methods have been examined in patients with sepsis, but they have not been extensively examined in patients with concurrent heart disease.

- False-positive SV variations in right ventricle dysfunction.

It is also possible to use continuous wave Doppler (CW) in VTI assessment. This method is particularly 
512 Wojciech Mielnicki et al Utility of transthoracic echocardiography (TTE) in assessing fluid responsiveness in critically ill patients

useful in the small and hyperdynamic left ventricle, when we suspect functional obstruction of LVOT. First of all, it is important to rule out any pathology in the LVOT tract causing anatomical obstruction (i.e hypertrophic cardiomyopathy, restrictive diseases). All these pathologies can generate high flow velocities in the left ventricle. Examination should start with PW Doppler, and after finding high velocities in the left ventricle (i.e. $>1.5 \mathrm{~m} / \mathrm{s}$ ), CW Doppler is placed parallel to the LVOT. High velocities in LVOT in the small and hyperdynamic left ventricle might suggest fluid responsiveness. Unfortunately, there is limited data in literature supporting high velocities in LVOT as a predictor of fluid responsiveness.

\section{Suggestions:}

a. Differences in VTI $\geq 20 \%$ in mechanically ventilated patients correlate well with fluid responsiveness.

b. Differences in LVOT maximal velocity $\geq 12 \%$ in mechanically ventilated patients in septic shock correlate well with fluid responsiveness.

c. Remember the important limitations of the technique.

\section{Passive Leg Raising (PLR)}

Passive leg raising is a good alternative to fluid bolus in patients suspected of hypovolemia [29,30]. During this test about $300 \mathrm{ml}$ of blood is mobilised from lower extremities and is transported quickly to the heart. PLR is safe for the patient and completely reversible.

At the beginning of this test, the patient is in $45^{\circ}$ semi-recumbent position and then lowered to horizontal plane with legs raised to $45^{\circ}$. In PLR we measure VTI in semi-recumbent position and again in horizontal plane with legs raised, about one minute after changing the patient's position. The biggest advantage of PLR is that it can be performed both in spontaneously breathing and mechanically ventilated patients $[29,31,32]$. It can also be performed in patients with arrhythmias [32]. Arrhythmias predispose to huge changes in VTI and it is absolutely necessary to take the sequence of at least five VTI measurements for the result to be credible. Increase in VTI of at least $12 \%$ in passive leg raising correlates very well with fluid responsiveness [32] (fig 7).

The PLR test has some important limitations [25,33]:

- Keeping the right alignment between the PW Doppler line and LVOT might be very difficult after leg raising.

- This test is unreliable in case of abdominal hypertension, when compression stockings are used or in severe hypovolemia when there is not enough blood in the legs to cause changes in VTI.

- In patients after surgery it might be impossible to perform PLR due to pain (e.g. multiple trauma),

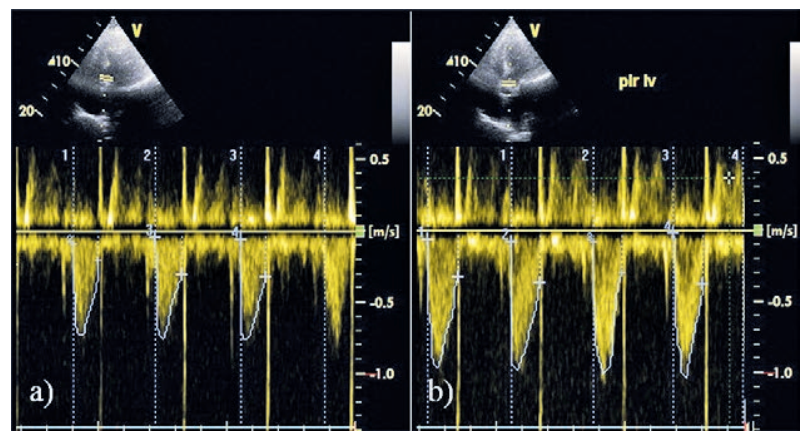

Fig 7. After performing the passive leg raising test manoeuvre there is considerable increase in the velocity-time integral which suggests fluid responsiveness. On the left: three measurements of VTI before the passive leg raising manoeuver; on the right: increase in VTI of more than $12 \%$ approximately one minute after PLR was performed strongly suggesting fluid-responsivness.

risk of aspiration (e.g. abdominal surgeries) or increase in intracranial pressure (e.g. head trauma or neurosurgeries).

- The technique is cumbersome and needs to be repeated.

Suggestions:

a. PLR can be used in spontaneously breathing and mechanically ventilated patients.

b. PLR is valid both in patients with sinus rhythm and arrhythmias.

c. Increase in VTI $\geq 12 \%$ correlates very well with fluid responsiveness.

d. In the case of an increase in VTI during PLR that is less than $12 \%$, the test remains inconclusive and it necessary to look for other signs and symptoms of hypovolemia.

e. In the case of a decrease in VTI during PLR, it is necessary to exclude improper probe alignment. Lower VTI after image optimisation can mean harmful effect of fluid infusion.

f. Remember the test limitations.

\section{Conclusion}

Transthoracic echocardiography is a good tool to assess fluid responsiveness in the critically ill patient. The most important test to predict fluid responsiveness is passive leg raising that can be reliably performed both in spontaneously breathing and mechanically ventilated patients. Inferior vena cava "distensibility index" and VTI variation assessment can only be performed in mechanically ventilated patients in a controlled mode. Although the role of echocardiography in a critical care setting is increasing, one has to remember the limitations of the technique. 


\section{References:}

1. Smith SH, Perner A. Higher vs. lower fluid volume for septic shock: clinical characteristics and outcome in unselected patients in a prospective, multicenter cohort. Crit Care 2012;16:R76.

2. Boyd JH, Forbes J, Nakada T, Walley KR, Russell JA. Fluid resuscitation in septic shock: a positive fluid balance and elevated central venous pressure are associated with increased mortality. Crit Care Med 2011;39:259-265.

3. Dellinger RP, Levy MM, Rhodes A, et al. Surviving sepsis campaign: international guidelines for management of severe sepsis and septic shock: 2012. Crit Care Med 2013;41:580-637.

4. Michard F, Teboul JL. Predicting fluid responsiveness in ICU patients: a critical analysis of the evidence. Chest 2002;121:2000-2008.

5. Slama M, Masson H, Teboul JL, et al. Respiratory variations of aortic VTI: a new index of hypovolemia and fluid responsiveness. Am J Physiol Heart Circ Physiol 2002;283:H1729-H1733.

6. de Backer D, Cholley BP, Slama M, Vieillard-Baron A, Vignon P. Editors. Hemodynamic Monitoring Using Echocardiography in the Critically Ill. Berlin, Heidelberg: Springer Berlin Heidelberg, 2011.

7. Vermeiren GL, Malbrain ML, Walpot JM. Cardiac Ultrasonography in the critical care setting: a practical approach to asses cardiac function and preload for the 'non-cardiologist'. Anaesthesiol Intensive Ther 2015;47:s89-s104.

8. Poelaert J. Assessment of loading conditions with cardiac ultrasound. A comprehensive review. Anaesthesiol Intensive Ther 2015;47:464-470.

9. Cheifetz IM. Cardiorespiratory interactions: the relationship between mechanical ventilation and hemodynamics. Respir Care 2014;59:1937-1945.

10. Robotham JL, Lixfeld W, Holland L, et al. The effects of positive end-expiratory pressure on right and left ventricular performance. Am Rev Respir Dis 1980;121:677-683.

11. Buda AJ, Pinsky MR, Ingels NB, Daughters GT 2nd, Stinson EB, Alderman EL. Effect of intrathoracic pressure on left ventricular performance. N Engl J Med 1979;301:453459.

12. Pinsky MR, Summer WR, Wise RA, Permutt S, Bromberger-Barnea B. Augmentation of cardiac function by elevation of intrathoracic pressure. J Appl Physiol Respir Environ Exerc Physiol 1983;54:950-955.

13. McGregor M. Current concepts: pulsus paradoxus. N Engl J Med 1979;301:480-482.

14. Feissel M, Michard F, Faller JP, Teboul JL. The respiratory variation in inferior vena cava diameter as a guide to fluid therapy. Intensive Care Med 2004;30:1834-1837.

15. Cole GD, Dhutia NM, Shun-Shin MJ, et al. Defining the real-world reproducibility of visual grading of left ventricular function and visual estimation of left ventricular ejection fraction: impact of image quality, experience and accreditation. Int J Cardiovasc Imaging 2015;31:13031314.
16. Tavernier B, Makhotine O, Lebuffe G, Dupont J, Scherpereel P. Systolic pressure variation as a guide to fluid therapy in patients with sepsis-induced hypotension. Anesthesiology 1998;89:1313-1321.

17. Charron C, Fessenmeyer C, Cosson C, et al. The influence of tidal volume on the dynamic variables of fluid responsiveness in critically ill patients. Anesth Analg 2006;102:1511-1517.

18. Walker CM, Reddy GP, Mohammed TL, Chung JH. Systolic anterior motion of the mitral valve. J Thorac Imaging 2012;27:W87.

19. Airapetian N, Maizel J, Alyamani O, et al. Does inferior vena cava respiratory variability predict fluid responsiveness in spontaneously breathing patients? Crit Care 2015;19:400.

20. Muller L, Bobbia X, Toumi M, et al. Respiratory variations of inferior vena cava diameter to predict fluid responsiveness in spontaneously breathing patients with acute circulatory failure: need for a cautious use. Crit Care. 2012;16:R188.

21. Kimura BJ, Dalugdugan R, Gilcrease GW 3rd, Phan JN, Showalter BK, Wolfson T. The effect of breathing manner on inferior vena caval diameter. Eur J Echocardiogr 2011;12:120-123.

22. Barbier C, Loubières $Y$, Schmit $C$, et al. Respiratory changes in inferior vena cava diameter are helpful in predicting fluid responsiveness in ventilated septic patients. Intensive Care Med 2004;30:1740-1746.

23. Marik PE, Cavallazzi R, Vasu T, Hirani A. Dynamic changes in arterial waveform derived variables and fluid responsiveness in mechanically ventilated patients: a systematic review of the literature. Crit Care Med 2009;37:2642-2647.

24. Feissel M, Michard F, Mangin I, Ruyer O, Faller JP, Teboul JL. Respiratory changes in aortic blood velocity as an indicator of fluid responsiveness in ventilated patients with septic shock. Chest 2001;119:867-873.

25. De Backer D, Fagnoul D. Intensive care ultrasound: VI. Fluid responsiveness and shock assessment. Ann Am Thorac Soc 2014;11:129-136.

26. Heenen S, De Backer D, Vincent JL. How can the response to volume expansion in patients with spontaneous respiratory movements be predicted? Crit Care 2006;10:R102.

27. De Backer D, Heenen S, Piagnerelli M, Koch M, Vincent JL. Pulse pressure variations to predict fluid responsiveness: influence of tidal volume. Intensive Care Med 2005;31:517-523.

28. Mahjoub Y, Pila C, Friggeri A, et al. Assessing fluid responsiveness in critically ill patients: False-positive pulse pressure variation is detected by Doppler echocardiographic evaluation of the right ventricle. Crit Care Med 2009;37:2570-2575.

29. Maizel J, Airapetian N, Lorne E, Tribouilloy C, Massy Z, Slama M. Diagnosis of central hypovolemia by using passive leg raising. Intensive Care Med 2007;33:1133-1138.

30. Monnet X, Rienzo M, Osman D, et al. Passive leg raising predicts fluid responsiveness in the critically ill. Crit Care Med 2006;34:1402-1407. 
514 Wojciech Mielnicki et al Utility of transthoracic echocardiography (TTE) in assessing fluid responsiveness in critically ill patients

31. Lamia B, Ochagavia A, Monnet X, Chemla D, Richard C, Teboul JL. Echocardiographic prediction of volume responsiveness in critically ill patients with spontaneously breathing activity. Intensive Care Med 2007;33:1125-1132.

32. Cavallaro F, Sandroni C, Marano C, et al. Diagnostic accuracy of passive leg raising for prediction of fluid respon- siveness in adults: systematic review and meta-analysis of clinical studies. Intensive Care Med 2010;36:1475-1483.

33. Mahjoub Y, Touzeau J, Airapetian N, et al. The passive legraising maneuver cannot accurately predict fluid responsiveness in patients with intra-abdominal hypertension. Crit Care Med 2010;38:1824-1829. 